\title{
A $\mathrm{Xe}^{+}$Plasma FIB Milling and Lift-out Approach for Site-specific Preparation of Large Volume Blocks for 3D-EBSD
}

\author{
B. Winiarski ${ }^{1,2}$, T.L. Burnett ${ }^{1}$, and P.J. Withers ${ }^{1}$ \\ 1. Henry Moseley X-ray Imaging Facility, School of Materials, University of Manchester, Manchester, \\ M13 9PL, U.K. \\ 2. FEI Company, Achtseweg Noord 5, Bldg 5651 GG, Eindhoven, The Netherlands
}

Three-dimensional electron backscatter diffraction (3D-EBSD) has emerged as a powerful technique for generating 3D crystallographic information of a microstructure at the micro to nanoscale [1]. Typically the technique uses a focused $\mathrm{Ga}^{+}$ion beam (FIB) for high precision ( $>10 \mathrm{~nm}$ per slice) serial sectioning generating consecutive ion milled cross sections with each milled surface subsequently mapped using EBSD. The successive EBSD maps are combined using a suitable post-processing method to generate a crystallographic volume of the microstructure [1] and subsequent analyses, e.g. grain size, grains and grain boundary orientation statistics, etc. A principal limitation of $\mathrm{Ga}^{+}$FIBs in their application to typical engineering or industrial applications is low milling rates of various materials irradiated with $\mathrm{Ga}+$ ions and maximum FIB current of $60 \mathrm{nA}$ [2], practically limiting the volume of excavated material to about $(50 \mu \mathrm{m})^{3}$ this is problematic as many engineering materials have grain size many 10's of microns which this technique cannot meaningfully investigate. Emerging dual beam $\mathrm{Xe}^{+}$Plasma FIBSEM (PFIB-SEM) systems not only allows removal rates some 20-50× faster [2], but also appear to generate less damage (amorphisation, implantation, phase transformation, etc.) than $\mathrm{Ga}^{+}$ions [2]. Volumes some 1000× greater and many times deeper than can be accessed using PFIB dual beam microscopes and promise to radically extend our capability for 3D tomography, 3D EDX, 3D EBSD as well as correlative tomography [3]. Here we examine the potential of $\mathrm{Xe}^{+}$PFIB-SEM for site-specific preparation of large volume blocks $\left(250 \times 150 \times 150 \mu^{3}\right)$ for 3D-EBSD of shot-peened AA7075 aluminum alloy below sharp notches [4] and around a stress corrosion crack tip in AA7032 aluminum alloy [5].

As a practical example in this study we used two aluminum alloys, AA7075 and AA7032. The material volumes for subsequent EBSD study are situated in locations typically not accessible by conventional 3D-EBSD: the notch tip of shot-peened 7075 and stress corrosion crack tip extracted from a compact tension specimen with the region of interest identified in using X-Ray micro Computed Tomography [4]. Sample preparation is presented elsewhere [4, 5]. For the site-specific preparation of large volume blocks we used FEI Helios $\mathrm{Xe}^{+}$Plasma Focused Ion Beam - SEM microscope equipped with Pt metalorganic gas injection system (GIS) and FEI EasyLift ${ }^{\mathrm{TM}}$ micromanipulator. The samples were mounted on $45^{\circ}$ tilt sample holders. Excavated blocks were mounted to standard TEM grid attached using sliver dag to 54 degree face of EBSD sample holder.

We have developed the following workflow to enable these type of measurements: (a) After finding the specific location a $4 \mu \mathrm{m}$ thick Pt protective layer of $250 \times 150 \mu \mathrm{m}^{2}$ (Figure 1a) was sputtered on the top surface using gas injection system (GIS) and PFIB; (b) Large side trenches with a lamella-like ligament on the left side of the block were milled at normal angle to the top surface near the Pt cup using $1.3 \mu \mathrm{A} @ 30 \mathrm{kV}$ (Figure 1a), regular (RCS) and cleaning cross-section (CCS); (c) A back trench was milled using the same PFIB settings but at $88^{\circ}$ angle to the top surface (Figure 1a). This allowed obtaining right angle between the top surface and the back face; (d) Subsequently, the sample was rotated by $180^{\circ}$ and the bottom face of the block and a thin ligament was milled using the same PFIB settings at angle about $2^{\circ}$ to the top surface (Figure 1b); (e) The stage was tilted to $45^{\circ}$, so the top 
surface of block is positioned horizontally. EasyLift ${ }^{\mathrm{TM}}$ needle was inserted, positioned in the top-right corner of the block (away from the ligament) and attached to the block using Pt layer (Figure 1c); (f) GIS was retracted, the ligament was milled away using $30 \mathrm{kV} @ 180 \mathrm{nA}$, and the needle with attached block was retracted to park position; (g) the chamber was vented, EBSD sample holder with TEM grid was positioned on the stage and the chamber was pumped; (h) before inserting the needle the stage was tilted to $38^{\circ}$, thus the TEM grid is positioned vertically (Figure $1 \mathrm{~d}-\mathrm{f}$ ); (i) EasyLift ${ }^{\mathrm{TM}}$ positioned the block against TEM grid post and sample was attached using Pt ligaments sputtered at the top and side of block (Figure 1 e); (j) the needle was detached from the block using CCS and 59 nA @ 30 kV (Figure 1 f); (k) Finally we used FEI EBS3 and Oxford Instruments HKL Fast Acquisition packages for automated 3D-EBSD data acquisition.

Presented procedure has a several benefits: (i) $45^{\circ}$-tilt sample holder allows milling the block from two orthogonal directions (ii) the block face is well defined; (iii) the signal is equally accessible for detection during milling and acquisition; (iv) lack of shadowing effects of the block face from the sides and the bottom of block.

We demonstrated the feasibility of site-specific preparation and lift-out workflow of large volume blocks for 3D-EBSD using FEI Helios $\mathrm{Xe}^{+}$PFIB-SEM microscope. This procedure has a great potential to expand our capabilities for PFIB-SEM high-resolution serial sectioning tomography and 3D-EDX element mapping.

References:

[1] Borgh I, Hedstrom P, Odqvist J, et al. Acta Materialia 13(2013): p. 4726-4733

[2] Burnett TL, Kelley R, Winiarski, B, at al. Ultramicroscopy 161(2016): p. 119-129

[3] Burnett TL, ; McDonald, SA, Gholinia A, et al. Scientific Reports 4(2014): 4711

[4] Winiarski B, Benedetti M, Fontanari V, et al., Vol. 9 Book Series: Conference Proceedings of the SEM Series, Springer, (2016): p. 215-223

[5] Burnett TL, Holroyd NJH, Scamans GM, et al. Corrosion Reviews Vol. 33, 6(2015): p. 443-454
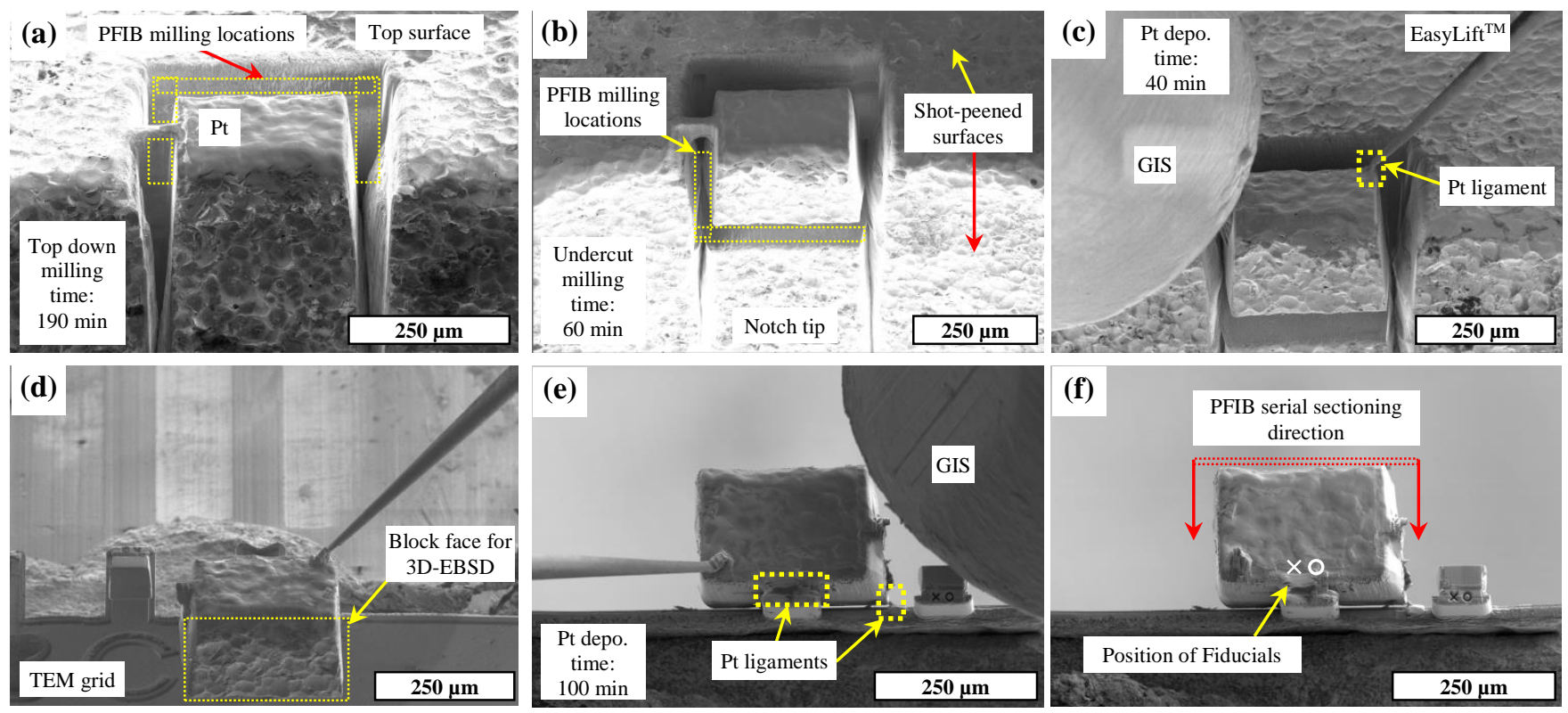

Figure 1. Shows site-specific sample block preparation of shot-peened 7075 with a notch (a-b) and liftout procedure (c-f). (a, b, e, f) SEM images (5 kV @ 1.4 nA ETD SE); (c, d) PFIB images (30 kV@ $24 \mathrm{pA}, \mathrm{ICE})$. 